\title{
Local changes in computational non-rapid eye movement sleep depth in infants
}

\section{Satomaa, Anna-Liisa}

2018-02

Satomaa , A-L , Saarenpaa-Heikkila , O , Huupponen , E, Kirjavainen , T, Heinonen , J \& Himanen, S-L 2018 , ' Local changes in computational non-rapid eye movement sleep depth in infants ' , Clinical Neurophysiology , vol. 129 , no. 2 , pp. 448-454 . https://doi.org/10.1016/j.clinph.2017.09.116

http://hdl.handle.net/10138/301080

https://doi.org/10.1016/j.clinph.2017.09.116

publishedVersion

Downloaded from Helda, University of Helsinki institutional repository.

This is an electronic reprint of the original article.

This reprint may differ from the original in pagination and typographic detail.

Please cite the original version. 


\title{
Local changes in computational non-rapid eye movement sleep depth in infants
}

\author{
Anna-Liisa Satomaa ${ }^{\mathrm{a}, *}$, Outi Saarenpää-Heikkilä ${ }^{\mathrm{b}}$, Eero Huupponen ${ }^{\mathrm{a}}$, Turkka Kirjavainen ${ }^{\mathrm{c}}$, \\ Juhani Heinonen ${ }^{\mathrm{d}}$, Sari-Leena Himanen ${ }^{\mathrm{a}, \mathrm{d}}$ \\ a Department of Clinical Neurophysiology, Tampere University Hospital, Medical Imaging Centre and Hospital Pharmacy, Pirkanmaa Hospital District, Tampere, Finland \\ ${ }^{\mathrm{b}}$ Tampere University Hospital, Department of Pediatrics, Tampere, Finland \\ ${ }^{\mathrm{c}}$ Helsinki University Hospital, Department of Pediatrics, Helsinki, Finland \\ ${ }^{\mathrm{d}}$ Faculty of Medicine and Life Sciences, University of Tampere, Tampere, Finland
}

\section{A R T I C L E I N F O}

\section{Article history:}

Accepted 24 September 2017

Available online 16 October 2017

\section{Keywords:}

Infant

Sleep

SWA

NREM

EEG

Maturation

Sleep depth

\section{H I G H L I G H T S}

- The EEG-based quantitative sleep depth analysis shows age-dependent changes in young infants.

- Young infants have local sleep depth differences.

- The local sleep depth differences probably correlate to cortical maturation.

\section{A B S T R A C T}

Objective: Deep NREM sleep and its hallmark EEG phenomenon slow wave activity (SWA) are under homeostatic control in adults. SWA is also locally regulated as it increases in the brain areas that have been used intensively. Moreover, in children, SWA is a marker of cortical maturation. In the present study the local properties of NREM sleep depth were evaluated using the quantitative mean frequency method. We aimed to study if age is related to NREM sleep depth in young infants. In addition, we studied if young infants have local differences in their NREM sleep.

Methods: Ambulatory over-night polysomnographies were recorded in 59 healthy and full-term infants at the age of one month. The infants were divided into two age groups ( $<44$ weeks and $\geq 44$ weeks) to allow maturational evaluations.

Results: The quantitative sleep depth analysis showed differences between the age groups. In addition, there were local sleep depth differences within the age groups.

Conclusions: The sleep depth change with age is most likely related to cortical maturation, whereas the local sleep depth gradients might also reflect the use-dependent properties of SWA.

Significance: The results support the idea that young infants have frontal cortical processing.

(c) 2017 International Federation of Clinical Neurophysiology. Published by Elsevier Ireland Ltd. All rights

reserved.

\section{Introduction}

Newborns and young infants spend most of their time sleeping, and the quality of sleep has been found to be important for their development. Although the contribution of each sleep stage is different, both REM and NREM sleep have important functional roles in brain maturation and in normal synaptic development (Graven, 2006). As for NREM, deep NREM is considered to be the most

* Corresponding author at: Department of Clinical Neurophysiology, Tampere University Hospital, Medical Imaging Centre and Hospital Pharmacy, Pirkanmaa Hospital District, Teiskontie 35, 33520 Tampere, Finland. Fax: +358 331164199.

E-mail address: anna-liisa.satomaa@pshp.fi (A.-L. Satomaa). important sleep stage for infants. In adults, slow wave sleep and slow wave activity (SWA) of NREM sleep are homeostatically regulated and considered a measure of sleep pressure (Borbély and Achermann, 1999). On the other hand, SWA is also a local phenomenon that is known to increase locally at the brain area that has been in intensive use or after learning a specific task (Kattler et al., 1994; Huber et al., 2004). Correspondingly, it has been shown that those cortical areas that have been used less present decreased SWA during subsequent sleep (Huber et al., 2006). These use-dependent features of SWA reflect the important function of NREM sleep in learning, synaptic plasticity, and in homeostatic brain restoration. Moreover, in children, SWA is considered to 
reflect brain maturation in terms of increased synaptic density and developing plasticity (Kurth et al., 2010). The maturation of the brain follows a posterior-anterior trend in that the occipital areas mature first and the frontal areas last (Kurth et al., 2010; Shaw et al., 2008). Furthermore, SWA has been shown to increase locally over maturing brain regions (Kurth et al., 2010), predicting the maturation of skills (Kurth et al., 2012). Accordingly, the local deep sleep and SWA properties of children have usually been studied in the context of cortical maturation with an emphasis on posterioranterior changes with age (Bosch-Bayard et al., 2012; Buchmann et al., 2011; Fattinger et al., 2014; Kurth et al., 2012). Even if there is a growing body of evidence that at least some sensory input is processed cortically in the frontal areas in a newborn (Sakatani et al., 1999; Sanders et al., 2002; Schriever et al., 2014; Taga et al., 2003; Zaramella et al., 2001), it has been presented that the frontal maximum of SWA does not evolve until adolescence (Kurth et al., 2010).

In the present study, we aim to find out if young infants have local differences in their NREM sleep EEG and if these local differences might support the idea of the supposed frontal cortical processing. Moreover, we aim to study if age is related to NREM sleep depth in the young infants.

\section{Methods}

As a part of the CHILD-SLEEP STUDY (Paavonen et al., 2017), a multidisciplinary project comprising 1671 infants born at Tampere University Hospital during 4/2011-2/2013, 88 ambulatory overnight polysomnographies (PSG) were recorded at the age of one month. The study protocol and the inclusion criteria for the PSG study are described in detail in our previous work (Satomaa et al., 2016). In short, the recruited infants were born full-term and healthy. The overnight PSGs were recorded with Embla Titanium equipment according to the usual bed time schedule of each family. A PSG technician visited the homes of each family to attach and remove the electrodes. The following signals were recorded: 6 channels of EEG (F4-, C4-, O2-A1; F3-, C3-, O1-A2), EOG (right and left), submental EMG, oxygen saturation (pulse oximeter, Nonin, with a two-beat averaging time) with waveform, thoracoabdominal inductance plethysmography, diaphragmatic and abdominal EMG, Emfit mattress sensor, ECG, and oronasal thermistor (Dymedix). The nasal pressure transducer was omitted from the protocol to minimize the sleep-disturbing effects of the recording equipment (Goodwin et al., 2001). The sampling rate for the EEG signals was $256 \mathrm{~Hz}$. The Ethical Committee of Pirkanmaa Hospital District and the City of Tampere approved the study (R11032). The parents of all the recruited infants gave their written informed consent.

\subsection{Participants}

In total, 88 healthy and full-term one-month-olds were recorded. The sleep parameters of 84 of these 88 infants are discussed in our recent work. In the present study, 29 recordings (33\%) were not included due to technical failures of one or more EEG channels. Therefore, the study sample comprised 59 infants (32 girls and 27 boys) whose conceptional age at birth was on average 40.1 weeks (38.6-41.7 weeks). At the time of the recording, the mean conceptional age of the infants was 44.6 weeks (42.4-48.4 weeks).

To evaluate the effect of age on the quality of sleep, the children were divided into two groups based on age at the time of recording. A cut-off point of one month ( 44 weeks) was chosen, because the same division had been used in the previous study on the same cohort of infants (Satomaa et al., 2016). The younger group comprised 21 infants (36\%) who were less than 44 weeks of age at the time of recording (younger group, mean age 43.2 weeks, range 42.4-43.9 weeks). Of these, 9 (43\%) were boys. The second group comprised 38 infants (64\%) who were 44 weeks of age or older at the time of recording (older group, mean age 45.5 weeks, range 44.0-48.4). Of these, 18 (47\%) were boys.

\subsection{Visual analysis}

The PSGs were scored into the sleep stages by two independent clinical neurophysiologists (ALS, SLH) with an agreement of $80.3 \%$ (range 72.2-90.0\%) and kappa score of 0.73 (range 0.58-0.86) indicating substantial agreement (Landis and Koch, 1977). The sleep stages were scored according to the AASM 2012 scoring manual (Berry et al., 2012) as closely as possible. However, due to the age-related sleep-EEG features of the infants, some modifications were implemented (see Satomaa et al., 2016). The infant's state was scored as active sleep (AS), quiet sleep (QS), indeterminate sleep (IS), or wakefulness (W). The cortical arousals were visually scored according to the current guidelines (Grigg-Damberger et al., 2007; Iber et al., 2007; IPWG, 2005). The respiratory events were scored according to the pediatric rules in the AASM manual (Berry et al., 2012; Iber et al., 2007). Because the visual scoring was completed in 2013, and because the same visual analysis was used in our previous work on the same cohort, the 2016 AASM rules (Berry et al., 2015) were not used. The numbers of each type of respiratory events divided by total sleep time (TST) were expressed as respective respiratory event indices $(\mathrm{n} / \mathrm{h})$.

\subsection{Computational sleep depth analysis}

In the present work, computational sleep depth was quantified by means of computational EEG mean frequency. The deeper the physiological sleep, the lower are the obtained mean frequency values and vice versa. (Huupponen et al., 2009, 2011; Penzel et al., 1991; Saastamoinen et al., 2006). First, mean frequency values were computed from each of the six EEG channels at 1-s timeresolution by applying the method described in our previous work (Huupponen et al., 2009, 2011). This computing step provided six overnight mean frequency curves with values ranging from 0.5 $\mathrm{Hz}$ to $30 \mathrm{~Hz}$.

Two types of computational parameters were then determined on the basis of the mean frequency curves. The combined QS + IS sleep of the one-month-olds was considered a precursor of NREM sleep and termed NotREM sleep. In the present study, only NotREM sleep was taken into account in the analyses. As the first parameter type, the median of the mean frequency values during the NotREM sleep time were extracted from each EEG channel. Then, each mean frequency curve (in its total length) was smoothed using a sliding 31-s long median filter as described previously (Huupponen et al., 2009, 2011; Saastamoinen et al., 2007). As the second parameter type, the deep sleep percentage (DS\%), providing the share (in percentage) of the NotREM time containing deeper sleep than the threshold $(2 \mathrm{~Hz}$ and $4 \mathrm{~Hz}$ ), was extracted from each smoothed mean frequency curve. The resulting deep sleep percentage parameters were denoted DS2\% and DS4\%, respectively.

\subsection{Statistics}

Statistical comparisons were performed with SPSS software (v 21). The data were tested for normal distribution with the Kolmogorov-Smirnov and Shapiro-Wilk tests, and all parameters were not normally distributed. The sleep depth parameters were compared between the groups with the nonparametric MannWhitney U-test. In addition, the local sleep depth parameters were studied within the groups. The left-to-right and anterior-posterior comparisons of sleep depth parameters within the age groups were 
performed with the nonparametric Wilcoxon Signed Ranks test. The multiple comparisons were Bonferroni-corrected. P-values $<0.05$ were considered statistically significant.

\section{Results}

The visually scored sleep EEG parameters did not differ between the two age groups (Table 1 ). The only statistically significant difference in the respiratory parameters was found in OAHI: the infants in the younger age group had statistically more obstructive apneas and hypopneas, although the obstructive events were very rare in both groups.

AS\%, QS\%, IS\% = the duration of each sleep state divided by total sleep time (TST). Awakening index and arousal index = the number of awakenings divided by TST and the number of awakenings + cor tical arousals divided by TST, respectively. AHI = number of all apneas and hypopneas $/ \mathrm{h}$. OAHI $=\mathrm{n}$ of obstructive and mixed apneas and hypopneas/h. ODI3 = Oxygen desaturation index, the number of oxygen desaturations $\geq 3 \%$ x 60/TST.

The computational sleep depth parameters, reflecting local sleep depth at the six different EEG locations, of both age groups are presented in Figs. 1-3. The older age group had statistically lower median sleep depth than the younger age group at every EEG channel during the NotREM time (Fig. 1). DS4\% was abundant in both groups; however, the older group presented higher DS4\% values than the younger group at F4-, C3- and 01-channels (Fig. 2). In addition, the older age group showed higher DS2\% values than the younger group at all EEG locations (Fig. 3). Moreover, the local sleep depth measures were studied within the age groups. Neither age group showed any inter-hemispheric differences in any of the computational sleep depth measures. Instead, anterior-posterior (AP) comparisons within the hemispheres revealed several significant differences.

In the younger age group, the median sleep depth was lowest occipitally in the left hemisphere, whereas no significant differences were obtained in the right (Fig. 1). In the older age group, the median sleep depth was lowest frontally in the right hemisphere, whereas no significant differences were found in the left. The amount of DS4\% did not show any significant AP differences in either of the groups (Fig. 2). In the younger age group, DS2\% was higher occipitally than centrally in the left hemisphere (01 vs. C3). In the right hemisphere, DS2\% was higher frontally than centrally (F4 vs. C4, Fig. 3). In the older age group, DS2\% was highest frontally in the right hemisphere.

The inter-group anterior-posterior differences in sleep depth were studied statistically within each hemisphere. This was performed by comparing the sleep depth parameters at each electrode position of the left hemisphere in the younger group to each electrode position of the left hemisphere in the older group. The same evaluation was performed on the right hemisphere. The resulting statistically significant parameters are presented in Table 2 . The absolute values of the tested parameters are presented in the Figs. 1-3.

\section{Discussion}

As in our previous study, the visually scored sleep stage percentages or arousal indices did not differ between the two age groups (Satomaa et al., 2016). However, the computational sleep depth analysis of NotREM sleep revealed several differences between the two age groups. Compared with the younger group, the older age group had significantly lower median depth and a higher amount of DS2\% at every EEG channel. In addition, this group presented more DS4\% frontally in the right and centrally and occipitally in the left hemisphere than the younger age group.

To date, there have only been a handful of studies on the maturational changes of slow EEG frequencies during NREM sleep in the neonatal age group. Topographic evaluations on very young infants are especially scarce even if multichannel recordings have been performed (Chu et al., 2014; Korotchikova et al., 2009; Mandelbaum et al., 2000; Novelli et al., 2016; Otero et al., 2011; Pereda et al., 2006). Moreover, the number of infants in these studies has often been 21 or fewer (Chu et al., 2014; de la Cruz et al., 2007; Fattinger et al., 2014; Jenni et al., 2004; Mandelbaum et al., 2000; Novelli et al., 2016). In addition, only short periods of sleep ranging from some minutes to sixty minutes have commonly been analyzed (Bosch-Bayard et al., 2012; Chu et al., 2014; de la Cruz et al., 2007; González et al., 2011; Korotchikova et al., 2009; Ktonas et al., 1995; Mandelbaum et al., 2000; Pereda et al., 2006; Sankupellay et al., 2011), and the evaluated periods have often represented only daytime sleep (Chu et al., 2014; Korotchikova et al., 2009; Mandelbaum et al., 2000; Otero et al., 2011; Pereda et al., 2006). To the best of our knowledge, the only multichannel work that has used whole night recordings in the infant age group is a study by Novelli and co-workers (2016). They studied the EEG power spectra of 39 children aged between 0 and 48 months at eleven scalp locations during NREM sleep, but only the first $60 \mathrm{~min}$ were taken into account. The youngest group comprised 14 infants ( $0-3$ months). They report that delta activity has a stable prevalence at occipital and parietal brain areas across the age groups. Two other quantitative maturational studies (Jenni et al., 2004; Sankupellay et al., 2011) of the newborn age group should also be mentioned, even though their focus was not topographical. Jenni et al. (2004) studied power spectrum at the C4-P4 EEG channel during over-night sleep. Seven infants were studied at two weeks of age and seven at two months of age. The

Table 1

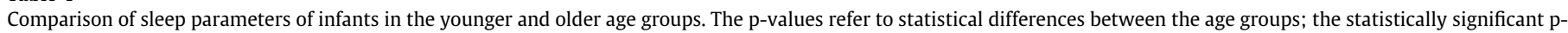
values are in italics and bolded.

\begin{tabular}{|c|c|c|c|c|c|c|c|}
\hline & \multicolumn{3}{|c|}{$\begin{array}{l}\text { Younger group } \\
<44 \text { weeks }(n=21)\end{array}$} & \multicolumn{3}{|c|}{$\begin{array}{l}\text { Older group } \\
\geq 44 \text { weeks }(n=38)\end{array}$} & \multirow[t]{2}{*}{$\mathrm{P}$} \\
\hline & Median & Range & SD & Median & Range & SD & \\
\hline Age (weeks at recording) & 43.1 & $42.4-43.9$ & 0.5 & 45.4 & $44.0-48.4$ & 0.9 & 0.000 \\
\hline TST (min) & 486.5 & $366.5-628$ & 60.9 & 496 & $380-592$ & 49.1 & 0.063 \\
\hline QS\% & 40.5 & $34.9-54.6$ & 4.5 & 41.2 & $30.3-57.2$ & 5.6 & 0.704 \\
\hline IS\% & 21.2 & $9.7-36.0$ & 6.2 & 21.0 & $12.5-35.4$ & 6.0 & 0.537 \\
\hline AS\% & 37.1 & $26.5-47.5$ & 6.7 & 36.6 & $27.5-54.1$ & 6.7 & 0.987 \\
\hline Awakening index $(\mathrm{n} / \mathrm{h})$ & 9.2 & $5.9-13.0$ & 1.9 & 7.9 & $4.8-13.3$ & 2.1 & 0.087 \\
\hline Arousal index $(\mathrm{n} / \mathrm{h})$ & 20.3 & $13.5-25.3$ & 3.0 & 18.0 & $12.1-29.0$ & 3.8 & 0.200 \\
\hline AHI (TST) (n/h) & 13.5 & $1.4-81.7$ & 19.3 & 8.5 & $0.5-63.8$ & 13.7 & 0.093 \\
\hline $\mathrm{CAI}(\mathrm{n} / \mathrm{h})$ & 11.6 & $1.4-79.8$ & 18.9 & 8.3 & $0.5-59.2$ & 13.0 & 0.226 \\
\hline OAHI (n/h) & 0 & $0-0.3$ & 0.1 & 0 & $0-0.0$ & 0.0 & 0.018 \\
\hline ODI3 (n/h) & 20.9 & $7.2-79.5$ & 21.8 & 15.1 & $0.6-75.5$ & 16.3 & 0.111 \\
\hline
\end{tabular}




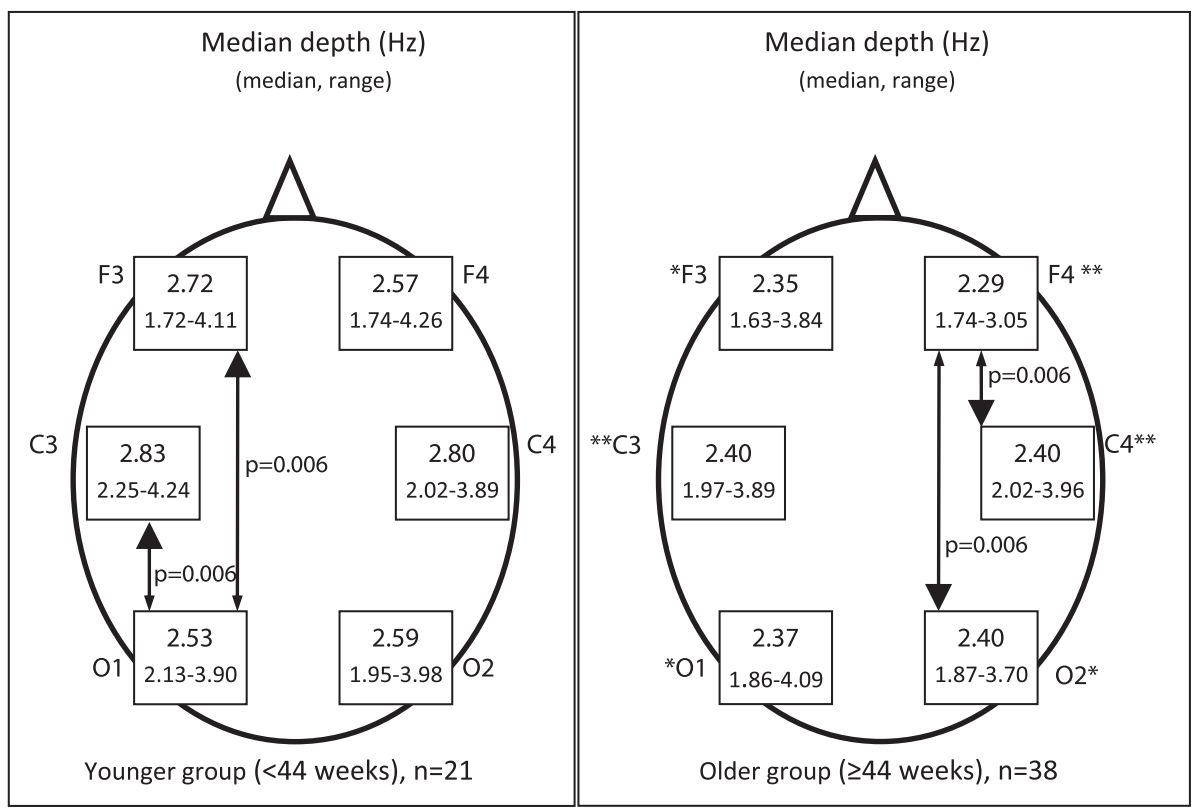

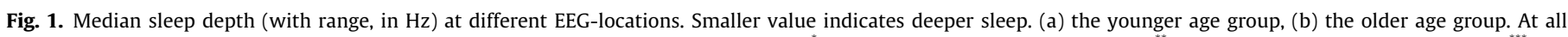

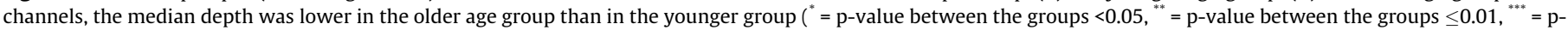
value between the groups $\leq 0.001$ ). The arrows denote the statistically significant anterior-posterior differences within the groups.

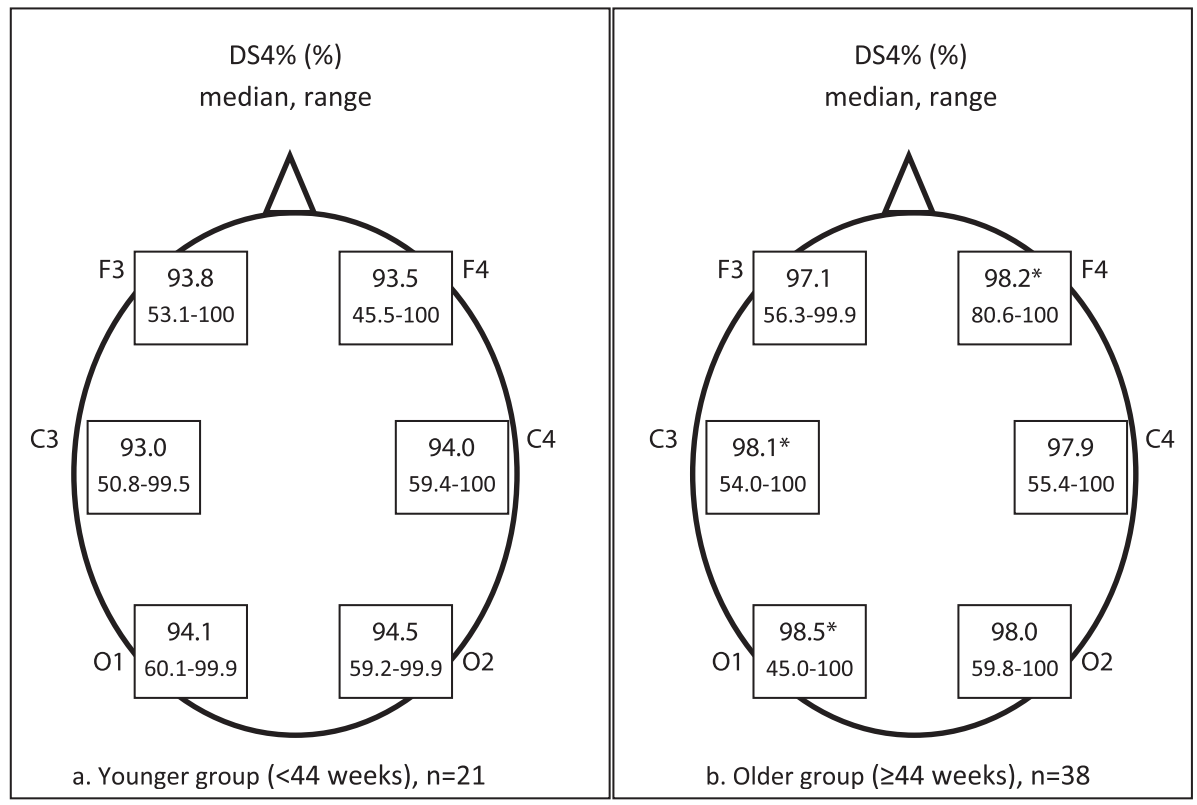

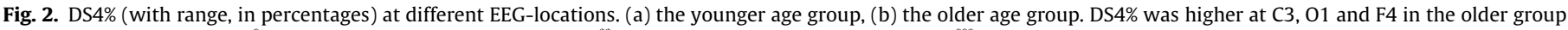

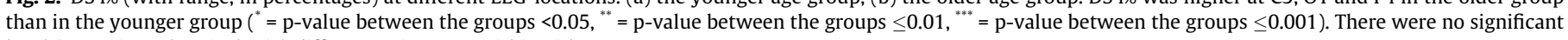
local (AP or inter-hemispheric) differences in DS4\% within either group.

power spectral changes in consecutive NREM cycles during the night were described. They found that at the age of two months the low frequency delta activity $(0.75 \mathrm{~Hz}-1.75 \mathrm{~Hz})$ presents an alternating pattern being high in every other NREM episode. Sankupellay et al. (2011) studied power spectrum at one central EEG channel during 15 min of nocturnal sleep. Of the 31 subjects studied, the youngest were two weeks of age, and the change in the power spectrum from two weeks to three months of age was described. Delta bandwidth power was found to dominate and increase during QS/N3 NREM from 2 weeks of age to two years.
Because the methods, subjects' age, EEG sample selection, and the electrode set-up varied substantially in the above mentioned studies, the general view of the quantitative slow EEG properties in newborns is rather fragmentary. A consistent finding, however, is that most of a newborn's sleep EEG power is in the delta range (González et al., 2011; Korotchikova et al., 2009; Mandelbaum et al., 2000; Pereda et al., 2006; Sankupellay et al., 2011). This finding was also evident in our study. It has been reported that delta activity increases during early infancy (Chu et al., 2014; Jenni et al., 2004; Pereda et al., 2006; Sankupellay et al., 2011), and the 


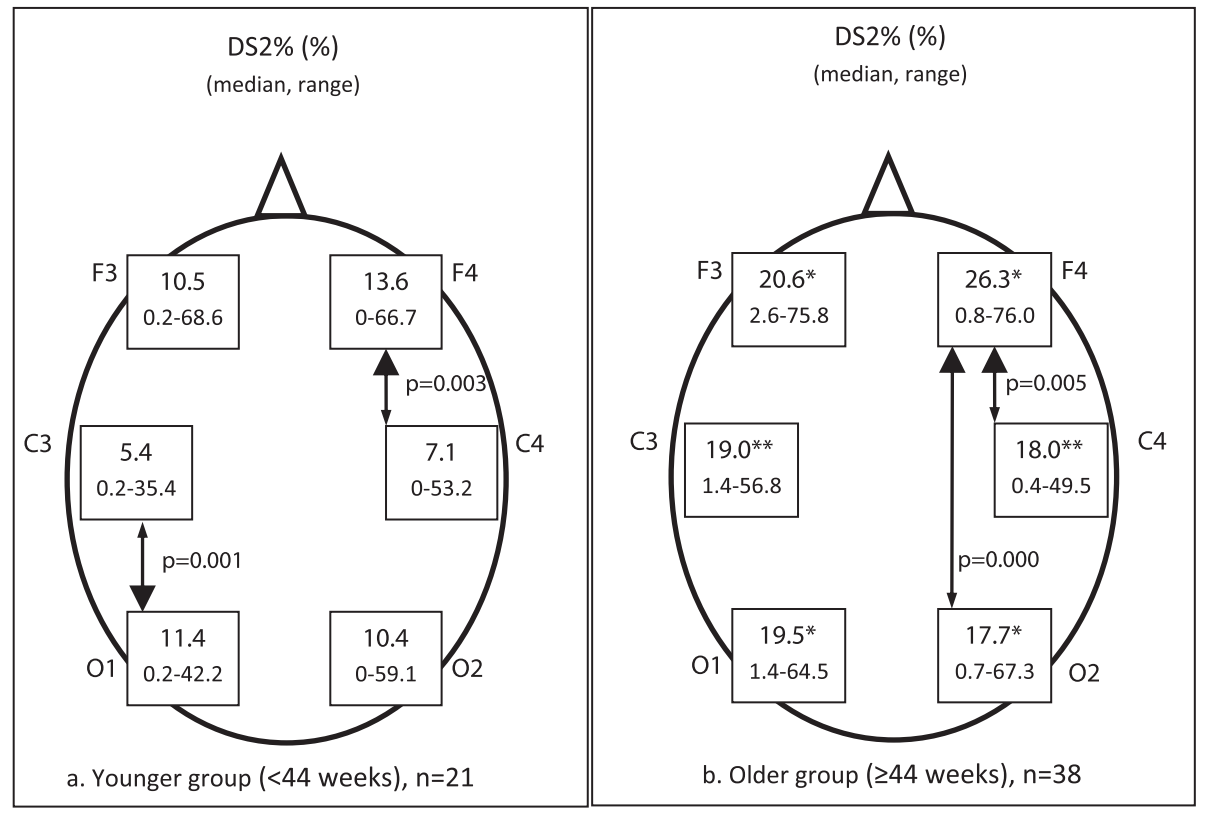

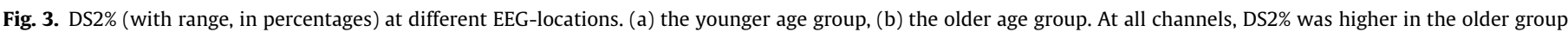

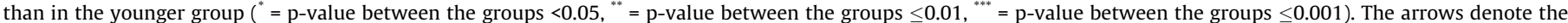
statistically significant anterior-posterior differences within the groups.

Table 2

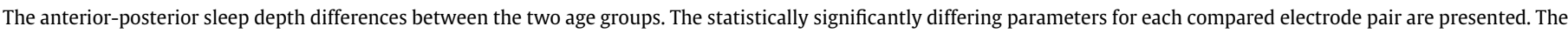
absolute values of these parameters are presented in the Figs. 1-3. (med = median sleep depth).

\begin{tabular}{|c|c|c|c|c|c|c|c|c|c|}
\hline & & \multicolumn{3}{|l|}{ Younger group } & & & \multicolumn{3}{|l|}{ Younger group } \\
\hline & & F3 & $\mathrm{C} 3$ & 01 & & & $\mathrm{~F} 4$ & $\mathrm{C} 4$ & $\mathrm{O} 2$ \\
\hline \multirow[t]{3}{*}{ Older group } & F3 & Med, DS4\%, DS2\% & Med, DS2\% & Med & Older group & $\mathrm{F} 4$ & Med, DS4\%, DS2\% & Med, DS4\%, DS2\% & Med, DS4\%, DS2\% \\
\hline & $\mathrm{C} 3$ & Med, DS4\%, DS2\% & Med, DS4\%, DS2\% & & & $\mathrm{C} 4$ & Med & Med, DS2\% & Med, DS4\%, DS2\% \\
\hline & 01 & Med, DS4\%, DS2\% & Med, DS4\%, DS2\% & Med, DS4\%, DS2\% & & $\mathrm{O} 2$ & Med & Med, DS2\% & Med, DS2\% \\
\hline
\end{tabular}

increase is generally believed to be related to cortical maturation. The results of our study (DS2\% was higher and median depth lower at every EEG location in the older age group) confirm that low EEG frequencies also increase with age in young infants, which in turn supports the previous idea that the increase in delta range EEG activity could be related to cortical maturation processes such as an increase in synaptic density (Buchmann et al., 2011; Huber and Born, 2014).

Whereas DS2\% and median sleep depth changed significantly with age at every EEG channel, DS4\% revealed more localized differences between the age groups. In the left posterior and central regions of the older age group, DS4\% was higher than in the younger group. The posterior enhancement of delta sleep with age is consistent with other studies on topographical maturationrelated changes of delta (Chu et al., 2014; Kurth et al., 2010, 2012; Novelli et al., 2016). In one study, however, delta activity was found to increase from preterm to about one month of age only at the left central but not the occipital area (Pereda et al., 2006). The methodological differences between that and our study (daytime nap versus whole NREM sleep, number of subjects) may account for the slightly different results.

The brain areas that are currently maturing present more SWA than the rest of the cortex (Kurth et al., 2012). Therefore, our finding that the older group had higher DS4\% than the younger group frontally in the right hemisphere might suggest that frontal cortical maturation is already ongoing during the first month of life. This finding differs from most of the previous studies in infants (Bosch-Bayard et al., 2012; Chu et al., 2014; Fattinger et al.,
2014; Mandelbaum et al., 2000; Novelli et al., 2016; Otero et al., 2011; Pereda et al., 2006). However, despite having a different study paradigm from ours, the frontal region also stood out in a study by González and co-workers (2011). They studied a total of 21 infants aged 39-45 weeks. The infants were further divided into three age groups each comprising seven children (39-40 weeks; 44-45 weeks). Intra and inter hemispheric functional connectivity was studied during AS and QS. Regarding QS, González and coworkers (2011) found that the inter-hemispheric coupling in the delta band progressively changed with age at the frontopolar and central regions.

On the other hand, it is also known that the brain areas that have been in intensive use during wakefulness present augmented SWA during subsequent sleep (Kattler et al., 1994). As there is increasing evidence that at least some sensory input is processed cortically in a newborn - even some association cortices have been found to participate (Sakatani et al., 1999; Sanders et al., 2002; Schriever et al., 2014; Taga et al., 2003; Zaramella et al., 2001) perhaps the right frontal emphasis of DS4\% in the older group of infants in the present study can also be attributed to the usedependent local increment of SWA.

The local sleep depth properties, other than inter-hemispheric differences and age-related changes, have rarely been studied in young infants. In the study by Novelli and co-workers (2016), delta activity showed prevalence at posterior regions during QS. Similarly, in the study by Chu et al. (2014), slow frequencies were most abundant in the posterior regions during QS. Chu and colleagues studied about 27 infants in the $0-3$ months age group. Based on 
the findings of these previous studies, a posterior dominance of deep sleep would have been expected in the present study, too. Indeed, in the younger age group, the left hemisphere showed slower activity occipitally compared with the more anterior areas, and DS2\% was more abundant occipitally than centrally.

In the present study, the anterior-posterior differences were also studied between the age groups. In the left hemisphere, F3 and $\mathrm{C} 3$ electrodes of the younger group differed significantly from F3, C3, and 01 electrodes of the older group by all studied parameters - median sleep depth was lower and DS2\% and DS4\% higher in the older group. However, $\mathrm{O} 1$ electrode of the younger group differed significantly from 01 of the older group (median depth, DS2\%, DS4\%), but not from C3, and from F3 by median depth only. This emphasizes the finding of relative slowness of the left occipital area in the younger group, as it was slow enough not to differ globally from the more anterior electrodes of the older group.

The older age group presented relative slowness of the right frontal area when compared with more posterior areas. This gradient within the right hemisphere manifested in both median sleep depth values and DS2\% amounts. The younger age group also showed the relative local slowness of the right frontal area, but the difference was only significant when compared with the right central area and it only visualized in DS2\%. Right frontal area stands out also in the inter-group anterior-posterior comparisons. Whereas $\mathrm{C} 4$ and $\mathrm{O} 2$ of the younger group differed by all studied sleep depth parameters from F4, C4 and $\mathrm{O} 2$ of the older group, $\mathrm{F} 4$ of the younger group differed from $\mathrm{C} 4$ and $\mathrm{O} 2$ of the older group by median depth only. This supports the finding of relative slowness in the right frontal area already during the first month of life.

Although the myelination of the frontal cortex is generally assumed to take place late, there are a few reports of frontal cortical responses to sensory stimulation in newborns. For example, exposure to olfactory stimuli is known to induce a frontal EEG activation in infants (Sanders et al., 2002). Olfactory event-related potentials (ERPs) are recordable in the majority of cooperative infants, and they localize frontally (Schriever et al., 2014). Moreover, hemodynamic responses to visual (Taga et al., 2003) and auditory (Zaramella et al., 2001) stimulation have been found frontally in the neonatal period. Therefore, the local enhancement of slow activity in the right frontal area in the present study could also be use-dependent in origin.

The quantitative method used in this study evaluates the mean frequency of EEG. Thus, compared to the conventional SWA power spectrum analysis, the mean frequency method gives additional information about sleep EEG, and it describes a wider frequency range. Another quantitative approach, the period amplitude analysis, is also known to produce more detailed information about frequency and amplitude than the SWA method (Ktonas and Gosalia, 1981). Neither the SWA nor the mean frequency approach is immune to EEG artifacts, e.g., eye and body movement artifacts. However, contrary to the visual and inherently subjective artifact removal required in the SWA analysis, in the quantitative mean frequency method no preprocessing or artifact removal is required. The median operation is the most effective part in minimizing the effects of EEG artifacts (Huupponen et al., 2009). We have previously shown that mean frequency measure correlates well with visual sleep staging (Huupponen et al., 2009, 2011; Saastamoinen et al., 2006, 2007).

\section{Conclusion}

Even though conventional visual scoring did not show any differences between the younger and older age groups in this study, the quantitative sleep depth analysis of NotREM sleep changed with age. Moreover, the one month old infants showed local sleep depth differences, which also changed with age. Most of the agedependent sleep depth change probably relates to cortical maturation. Some of it, however, might also reflect a use-dependent local increase of sleep depth. The local findings also support the idea that even very young infants have frontal cortical activity.

\section{Acknowledgements}

The Child Sleep Consortium is funded by the Academy of Finland (\#134880, \#277557). This study was financially supported by Foundation for Pediatric Research, Arvo ja Lea Ylppö Foundation, The Medical Society of Tampere, the Competitive Research Financing of the Expert Responsibility area of Tampere University Hospital, grant nos. 9P013, 9R007, 9R044, 9S007, 9S058. AS has received grants from the Finnish Sleep Research Society and The Research Foundation of Pulmonary Diseases.

\section{Conflict of interest statement}

None of the authors have potential conflicts of interest to be disclosed.

\section{References}

Berry RB, Budhiraja R, Gottlieb DJ, Gozal D, Iber C, Kapur VK, Marcus CL, Mehra R, Parthasarathy S, Quan SF, Redline S, Strohl KP, Davidson Ward SL, Tangredi MM. Rules for scoring respiratory events in sleep: update of the 2007 AASM manual for the scoring of sleep and associated events. deliberations of the sleep apnea definitions task force of the american academy of sleep medicine. J Clin Sleep Med 2012:8:597-619.

Berry RB, Brooks R, Gamaldo CE, Harding SM, Lloyd RM, Marcus CL, Vaughn BV. For the American Academy of Sleep Medicine. The AASM manual for the scoring of sleep and associated events: rules, terminology and technical specifications, version 2.2. Darien, Illinois: American Academy of Sleep Medicine; 2015. $<$ www.aasmnet.org>.

Borbély A, Achermann P. Sleep homeostasis and models of sleep regulation. J Biol Rhythms 1999:14:559-68.

Bosch-Bayard J, Valdés-Sosa P, Fernandez t, otero G, Rivero B, Ricardo-Garcell J, González-Frankenberger B, Galán-García L, Fernandez-Bouzas A, AubertVasquez E, Lage-Castellanos A, Rodríguez-Valdés R. Harmony T. 3D statistical parametric mapping of quiet sleep EEG in the first year of life. Neuroimage 2012;59:3297-308.

Buchmann A, Ringli M, Kurth S, Schraerer M, Geiger A, Jenni O, Huber R. EEG sleep slow-wave activity as a mirror of cortical maturation. Cereb Cortex 2011;21:607-15.

Chu CJ, Leahy J, Pathmanathan J, Kramer MA, Cash SS. The maturation of cortical sleep rhythms and networks over early development. Clin Neurophysiol 2014;125(7):1360-70.

de la Cruz DM, Mañas S, Pereda E, Garrido JM, López S, De Vera L, González JJ. Maturational changes in the interdependencies between cortical brain areas of neonates during sleep. Cereb Cortex 2007:17(3):583-90.

Fattinger S, Jenni O, Schmidt B, Achermann P, Huber R. Overnight changes in the slope of sleep slow waves during infancy. Sleep 2014;37:245-53.

González JJ, Mañas S, De Vera L, Méndez LD, López S, Garrido JM, Pereda E. Assessment of electroencephalographic functional connectivity in term and preterm neonates. Clin Neurophysiol 2011;122(4):696-702.

Goodwin JL, Enright PL, Kaemingk KL, Rosen GM, Morgan WJ, Fregosi RF, Quan SF. Feasibility of using unattended polysomnography in children for researchreport of the Tucson children's assessment of sleep apnea study (TuCASA). Sleep 2001;24:937-44.

Graven S. Sleep and brain development. Clin Perinatol 2006;33:693-706.

Grigg-Damberger M, Gozal D, Marcus CL, Quan SF, Rosen CL, Chervin RD, Wise M, Picchietti DL, Sheldon SH, Iber C. The visual scoring of sleep and arousal in infants and children. J Clin Sleep Med 2007;3:201-40.

Huber R, Ghilardi M, Massimini M, Tononi G. Local sleep and learning. Nature 2004;430:78-81.

Huber R, Ghilardi MF, Massimini M, Ferrarelli F, Riedner BA, Peterson MJ, Tononi G. Arm immobilization causes cortical plastic changes and locally decreases sleep slow wave activity. Nat Neurosci 2006;9(9):1169-76.

Huber R, Born J. Sleep, synaptic connectivity, and hippocampal memory during early development. Trends Cogn Sci 2014;18(3):141-52.

Huupponen E, Saunamäki T, Saastamoinen A, Kulkas A, Tenhunen M, Himanen SL. Improved computational fronto-central sleep depth parameters show differences between apnea patients and control subjects. Med Biol Eng Comput 2009;47(1):3-10.

Huupponen E, Kulkas A, Saastamoinen A, Tenhunen M, Himanen SL. Identification of deep sleep and awake with computational EEG measures. J Med Syst 2011;35:1413-20. 
Iber C, Ancoli-Israel S, Chesson A, Quan S. For the American Academy of Sleep Medicine. The AASM manual for the scoring of sleep and associated events: rules, terminology and technical specifications, 1st ed. Westchester: IL: American Academy of Sleep Medicine; 2007.

Jenni OG, Borbély AA, Achermann P. Development of the nocturnal sleep electroencephalogram in human infants. Am J Physiol Regul Integr Comp Physiol 2004;286:R528-38.

Kattler H, Dijk D, Borbély A. Effect of unilateral somatosensory stimulation prior to sleep on the sleep EEG in humans. J Sleep Res 1994;3:159-64.

Korotchikova I, Connolly S, Ryan CA, Murray DM, Temko A, Greene BR, Boylan GB. EEG in the healthy term newborn within 12 hours of birth. Clin Neurophysiol 2009;120(6):1046-53.

Ktonas PY, Gosalia AP. Spectral analysis vs. period-amplitude analysis of narrowband EEG activity: a comparison based on the sleep delta-frequency band. Sleep 1981;4(2):193-206.

Ktonas PY, Fagioli I, Salzarulo P. Delta $(0.5-1.5 \mathrm{~Hz})$ and sigma $(11.5-15.5 \mathrm{~Hz})$ EEG power dynamics throughout quiet sleep in infants. Electroencephalogr Clin Neurophysiol 1995;95(2):90-6.

Kurth S, Ringli M, Geiger A, LeBourgeois M, Jenni O, Huber R. Mapping of cortical activity in the first two decades of life: a high density sleep electroencephalogram study. J Neurosci 2010;30:13211-9.

Kurth S, Ringli M, LeBourgeois ML, Geiger A, Buchmann A, Jenni O, Huber R. Mapping the electrophysiological marker of sleep depth reveals skill maturation in children and adolescents. Neuroimage 2012;63:959-65.

Landis JR, Koch GG. The measurement of observer agreement for categorical data. Biometrics 1977:33:159-74.

Mandelbaum DE, Krawciw N, Assing E, Ostfeld B, Washburn D, Rosenfeld D, Hiatt M, Hegyi T. Topographic mapping of brain potentials in the newborn infant: the establishment of normal values and utility in assessing infants with neurological injury. Acta Paediatr 2000;89(9):1104-10.

Novelli L, D’atri A, Marzano C, Finotti E, Ferrara M, Bruni O, De Gennaro L. Mapping changes in cortical activity during sleep in the first 4 years of life. J Sleep Res 2016;25(4):381-9.

Otero GA, Harmony T, Pliego-Rivero FB, Ricardo-Garcell J, Bosch-Bayard J, PorcayoMercado R, Fernández-Bouzas A, Díaz-Comas L, Galán L, Vieyra-Reyes P, Fernández T. QEEG norms for the first year of life. Early Hum Dev 2011;87 (10):691-703.

Paavonen J, Saarenpää-Heikkilä O, Pölkki P, Kylliäinen A, Porkka-Heiskanen T, Paunio T. Maternal and paternal sleep during pregnancy in the child-sleep birth cohort. Sleep Med 2017;29:47-56.
Penzel T, Stephan K, Kubicki S, Herrmann WM. Integrated sleep analysis, with emphasis on automatic methods. In: Degen R, Rodin EA, editors. Epilepsy, sleep and sleep deprivation. 2nd ed. Amsterdam: Elsevier Science Publishers; 1991. p. $177-200$.

Pereda E, de La Cruz D, Mañas S, Garrido J, López S, González J. Topography of EEC complexity in human neonates: effect of the postmenstrual age and the sleep state. Neurosci Lett 2006;394:152-7.

Saastamoinen A, Oja H, Huupponen E, Varri A, Hasan J, Himanen SL. Topographic differences in mean computational sleep depth between healthy controls and obstructive sleep apnoea patients. J Neurosci Methods 2006;157(1):178-84.

Saastamoinen A, Huupponen E, Värri A, Hasan J, Himanen S-L. Systematic performance evaluation of a continuous-scale sleep depth measure. Med Eng Phys 2007;29:1119-31.

Sakatani K, Chen S, Lichty W, Zuo H, Wang YP. Cerebral blood oxygenation changes induced by auditory stimulation in newborn infants measured by near infrared spectroscopy. Early Hum Dev 1999;55(3):229-36.

Sanders C, Diego M, Fernandez M, Field T, Hernandez-Reif M, Roca A. EEC asymmetry responses to lavender and rosemary aromas in adults and infants. Int J Neurosci 2002;112:1305-20.

Sankupellay M, Wilson S, Heussler H, Parsley C, Yuill M, Dakin C. Characteristics of sleep EEG power spectra in healthy infants in the first two years of life. Clin Neurophysiol 2011;122:236-43.

Satomaa AL, Saarenpää-Heikkilä O, Paavonen EJ, Himanen SL. The adapted American Academy of Sleep Medicine sleep scoring criteria in one month old infants: a means to improve comparability? Clin Neurophysiol 2016;127(2):1410-8.

Schriever VA, Góis-Eanes M, Schuster B, Huart C, Hummel T. Olfactory event-related potentials in infants. J Pediatr 2014;165(2):372-5.

Shaw P, Kabani NJ, Larch JP, Echstrand K, Lenroot R, Gogtay N, Greenstein D, Clasen L, Evans A, Rapaport JL, Giedd JN, Wise SP. Neurodevelopmental trajectories of the human cerebral cortex. J Neurosci 2008;28:3586-94.

Taga G, Asakawa K, Hirasawa K, Konishi Y. Hemodynamic responses to visual stimulation in occipital and frontal cortex of newborn infants: a near-infrared optical topography study. Early Hum Dev 2003;75(Suppl):S203-10.

The international pediatric work group on arousals. IPWG). The scoring of arousals in healthy term infants (between the ages of 1 and 6 months. J Sleep Res 2005; 14:37-41.

Zaramella P, Freato F, Amigoni A, Salvadori S, Marangoni P, Suppiej A, Schiavo B, Chiandetti L. Brain auditory activation measured by near-infrared spectroscopy (NIRS) in neonates. Pediatr Res 2001;49(2):213-9. 